\title{
MeV-range velocity-space tomography from gamma-ray and neutron emission spectrometry measurements at JET
}

Salewski, Mirko; Nocente, M.; Jacobsen, Asger Schou; Binda, F.; Cazzaniga, C.; Ericsson, G.; Eriksson, J.; Gorini, G.; Hellesen, C.; Hjalmarsson, A.

Total number of authors:

23

Published in:

Nuclear Fusion

Link to article, DOI:

10.1088/1741-4326/aa60e9

Publication date:

2017

Document Version

Early version, also known as pre-print

Link back to DTU Orbit

Citation (APA):

Salewski, M., Nocente, M., Jacobsen, A. S., Binda, F., Cazzaniga, C., Ericsson, G., Eriksson, J., Gorini, G., Hellesen, C., Hjalmarsson, A., Kiptily, V. G., Koskela, T., Korsholm, S. B., Kurki-Suonio, T., Leipold, F., Madsen, J., Moseev, D., Nielsen, S. K., Rasmussen, J., ... Tardocchi, M. (2017). MeV-range velocity-space tomography from gamma-ray and neutron emission spectrometry measurements at JET. Nuclear Fusion, 57(5), [056001 ]. https://doi.org/10.1088/1741-4326/aa60e9

\section{General rights}

Copyright and moral rights for the publications made accessible in the public portal are retained by the authors and/or other copyright owners and it is a condition of accessing publications that users recognise and abide by the legal requirements associated with these rights.

- Users may download and print one copy of any publication from the public portal for the purpose of private study or research.

- You may not further distribute the material or use it for any profit-making activity or commercial gain

- You may freely distribute the URL identifying the publication in the public portal 
This document is intended for publication in the open literature. It is made available on the clear understanding that it may not be further circulated and extracts or references may not be published prior to publication of the original when applicable, or without the consent of the Publications Officer, EUROfusion Programme Management Unit, Culham Science Centre, Abingdon, Oxon, OX14 3DB, UK or e-mail Publications.Officer@euro-fusion.org

Enquiries about Copyright and reproduction should be addressed to the Publications Officer, EUROfusion Programme Management Unit, Culham Science Centre, Abingdon, Oxon, OX14 3DB, UK or e-mail Publications.Officer@euro-fusion.org

The contents of this preprint and all other EUROfusion Preprints, Reports and Conference Papers are available to view online free at http://www.euro-fusionscipub.org. This site has full search facilities and e-mail alert options. In the JET specific papers the diagrams contained within the PDFs on this site are hyperlinked 


\title{
MeV-range velocity-space tomography from gamma-ray and neutron emission spectrometry measurements at JET
}

\author{
M. Salewski ${ }^{1}$, M. Nocente ${ }^{2,3}$, A.S. Jacobsen ${ }^{4}$, F. Binda ${ }^{5}$, \\ C. Cazzaniga $^{6}$, G. Ericsson ${ }^{5}$, J. Eriksson ${ }^{5}$, G. Gorini ${ }^{2,3}$, \\ C. Hellesen ${ }^{5}$, A. Hjalmarsson ${ }^{5}$, V.G. Kiptily ${ }^{7}$, T. Koskela ${ }^{8,9}$, \\ S.B. Korsholm ${ }^{1}$, T. Kurki-Suonio ${ }^{8}$, F. Leipold ${ }^{1}$, J. Madsen ${ }^{1}$, \\ D. Moseev $^{10}$, S.K. Nielsen ${ }^{1}$, J. Rasmussen ${ }^{1}$, M. Schneider ${ }^{11}$, \\ S.E. Sharapov ${ }^{7}$, M. Stejner ${ }^{1}, M$ Tardocchi $^{3}$ and JET \\ Contributors $\ddagger$ \\ EUROfusion Consortium, JET, Culham Science Centre, Abingdon, OX14 3DB, UK \\ 1 Technical University of Denmark, Department of Physics, Kgs. Lyngby, Denmark \\ ${ }^{2}$ University of Milano Bicocca, Department of Physics, Milano, Italy \\ ${ }^{3}$ Istituto di Fisica del Plasma, Consiglio Nazionale delle Ricerche, Milano, Italy \\ ${ }^{4}$ Max-Planck-Institut für Plasmaphysik, Garching, Germany \\ 5 Department of Physics and Astronomy, Uppsala University, Sweden \\ ${ }^{6}$ ISIS Facility, Science and Technology Facilities Council, Rutherford Appleton \\ Laboratory, Didcot, UK \\ 7 CCFE, Culham Science Centre, Abingdon, Oxon, UK \\ 8 Aalto University, Department of Applied Physics, Aalto, Finland \\ 9 NERSC, Lawrence Berkeley National Laboratory, Berkeley, CA, USA \\ 10 Max-Planck-Institut für Plasmaphysik, Greifswald, Germany \\ 11 ITER Organization, St Paul-lez-Durance Cedex, France \\ E-mail: msal@fysik.dtu.dk
}

\begin{abstract}
We demonstrate the measurement of a $2 \mathrm{D} \mathrm{MeV}$-range ion velocity distribution function by velocity-space tomography at JET. Deuterium ions were accelerated into the $\mathrm{MeV}$-range by third harmonic ion cyclotron resonance heating. We made measurements with three neutron emission spectrometers and a highresolution $\gamma$-ray spectrometer detecting the $\gamma$-rays released in two reactions. The tomographic inversion based on these five spectra is in excellent agreement with numerical simulations with the ASCOT-RFOF and the SPOT-RFOF codes. The length of the measured fast-ion tail corroborates the prediction that very few particles are accelerated above $2 \mathrm{MeV}$ due to the weak wave-particle interaction at higher energies.
\end{abstract}

‡ See the Appendix of "Overview of the JET results in support to ITER by X. Litaudon et al. to be published in Nuclear Fusion Special issue: overview and summary reports from the 26th Fusion Energy Conference (Kyoto, Japan, 17-22 October 2016) 


\section{Introduction}

The era of burning plasmas is approaching with the construction of the ITER tokamak [1]. The goal for ITER is a fusion power of $P_{\text {fus }}=500 \mathrm{MW}$ for an auxiliary heating power of $P_{\text {aux }}=50 \mathrm{MW}$ which gives a power amplification of $Q=P_{\text {fus }} / P_{\text {aux }}=10$ [2]. Many present designs for the next-step device DEMO hope for $Q=30-50[3,4]$. Such burning plasmas are predominantly self-heated by MeV-range $\alpha$-particles generated in the fusion reaction $\mathrm{D}(\mathrm{T}, \mathrm{n}) \alpha$. Since the fusion born $\alpha$-particle power is $P_{\alpha}=P_{\text {fus }} / 5$, the plasma self-heating fraction $\eta_{\alpha}$ is

$$
\eta_{\alpha}=\frac{P_{\alpha}}{P_{\alpha}+P_{\text {aux }}}=\frac{Q}{Q+5}
$$

The plasma self-heating fraction expresses the prominent role of the MeV-range $\alpha$ particles for the plasma heating [2]. The importance of plasma self-heating by $\alpha$-particles increases with $Q$ (figure 1). The fusion power world record discharge at JET in 1997 had $\eta_{\alpha}=11 \%(Q=0.64)$ just below 'break-even' at $\eta_{\alpha}=17 \%(Q=1)[5]$. Burning plasmas are predominantly self-heated by MeV-range alpha particles $\left(\eta_{\alpha}>50 \%, Q>5\right)$, as in ITER $\left(\eta_{\alpha}=67 \%\right)$ or DEMO $\left(\eta_{\alpha}=86-91 \%\right)$. At 'ignition' the plasma is completely self-heated $\left(\eta_{\alpha}=100 \%, Q \rightarrow \infty\right)$. Burning plasmas will offer new challenges since the temperature and density profiles are self-consistently determined by $\alpha$-particle heating rather than controlled by auxiliary heating. Furthermore, $\mathrm{MeV}$-range ions can drive a zoo of instabilities deteriorating the plasma performance [6-12]. Hence the development of diagnostics capable of measuring MeV-range ions is essential.

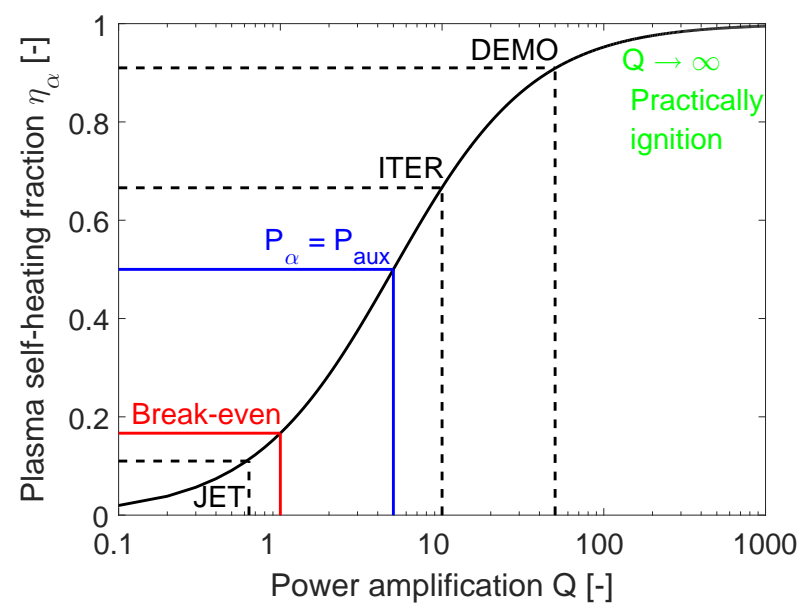

Figure 1. Plasma self-heating fraction $\eta_{\alpha}$ as function of the power amplification $Q$. Plasmas are predominantly heated by MeV-range alpha particles for $Q>5$.

$\mathrm{MeV}$-range ions are routinely generated by waves in the ion cyclotron range of frequencies (ICRF) and diagnosed by high-resolution $\gamma$-ray spectrometry (GRS) and neutron emission spectrometry (NES) at JET. Substantial densities of MeVrange $\alpha$-particles are expected in the upcoming DT campaign [13]. NES and GRS measurements can be related to energy spectra of neutrons and $\gamma$-rays, respectively 
[14-27]. Traditionally the measured 1D spectra are compared with simulated 1D spectra to obtain information about the fast ion phase-space distribution function. If the spectra agree, the simulation is thought to be a good model of the fast ions in the discharge. However, any disagreement is enormously difficult to interpret for several reasons. First, if several simultaneously acquired spectra are available, the amount of data one has to consider is rather large, often hundreds of data points. Second, each data point samples large regions of fast-ion phase space as illustrated by NES and GRS weight functions, and hence it is difficult to pinpoint the sources of any disagreement in phase space [28-31]. Third, the spectra also depend on nuisance parameters which are not of direct interest in fast-ion measurements, such as the thermal ion parameters.

Velocity-space tomography is a method to overcome these difficulties [32-43]. The method provides a measurement of the $2 \mathrm{D}$ velocity distribution function averaged over a spatial measurement volume. The rich and complex spectral measurement data are converted into $2 \mathrm{D}$ images of the velocity distribution function that are straightforward to interpret. The $2 \mathrm{D}$ images are the best useful fit to hundreds of simultaneously acquired data points, and nuisance parameters are accounted for. ASDEX Upgrade is equipped with collective Thomson scattering (CTS) and fast-ion $\mathrm{D}_{\alpha}$ (FIDA) spectroscopy diagnostics [44-48]. Velocity-space tomography is becoming a standard tool to visualize and analyze these FIDA and CTS measurements [35-43]. While FIDA spectroscopy works well for ion energies on the order of $100 \mathrm{keV}$, it suffers from the low charge-exchange cross sections at MeV-range energies [49-51]. Hence velocity-space tomography has so far been limited to ion energies below $120 \mathrm{keV}$. Here we demonstrate velocity-space tomography in the MeV-range based on GRS and NES at JET and measure a core-averaged 2D velocity distribution function of fast ions generated by third harmonic ICRF.

Section 2 describes the JET discharge, the diagnostic set-up and the NES and GRS measurements. In section 3 we discuss the velocity-space sensitivity of our detectors and how we select measurement data for the inversion. In section 4 we outline the velocity-space tomography approach. A measurement of a $2 \mathrm{D} \mathrm{MeV}$-range velocity distribution function and benchmarks with the ASCOT-RFOF codes [24,52] and the SPOT-RFOF [26] are presented in section 5. We discuss the potential and limitations of our approach in section 6 and draw conclusions in section 7 .

\section{NES and GRS measurements at JET}

Deuterium ions are routinely accelerated into the MeV-range by third harmonic ICRF heating [22-24]. The wave-particle interaction for third harmonic ICRF heating is much stronger for moderately energetic ions than for thermal ions [26]. Hence JET discharge \#86459 was heated by $4.5 \mathrm{MW}$ of neutral beam injection (NBI) with beam injection energies of $80 \mathrm{kev}, 100 \mathrm{keV}$ and $120 \mathrm{keV}$ providing the seed fast-ion population as well as $3 \mathrm{MW}$ of third harmonic ICRF heating accelerating the ions into the MeV range. As the central magnetic field was $B=2.25 \mathrm{~T}$, the ICRF wave frequency was $51 \mathrm{MHz}$. The 

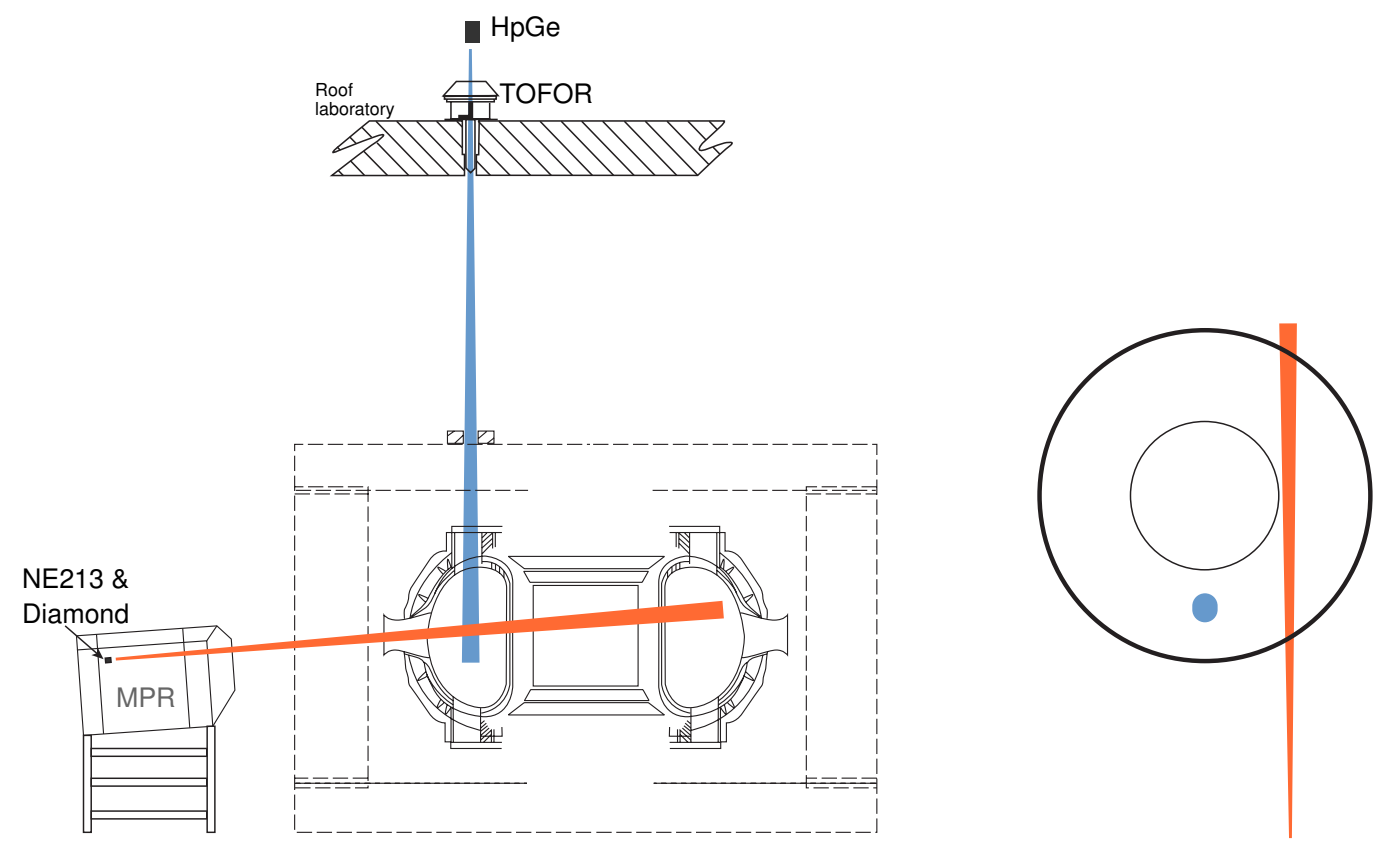

Figure 2. Cutaway view (left) and top view (right) of the lines-of-sight of the three neutron spectrometers (TOFOR, diamond, NE213) and the HpGe GRS detector. The angle between the lines-of-sight and the magnetic field is about $90^{\circ}$ for TOFOR and the HpGe detector and about $47^{\circ}$ for the compact diamond and NE213 spectrometers.

electron density was about $4 \times 10^{19} \mathrm{~m}^{-3}$.

The energetic deuterium tail formed in such plasma scenarios was simultaneously measured by three NES detectors and one high-purity Germanium (HpGe) GRS detector from $50.5 \mathrm{~s}$ to $52.1 \mathrm{~s}$ in discharge \#86459 (figure 2). The three neutron emission spectrometers detect neutrons with energies near $2.5 \mathrm{MeV}$ from the $\mathrm{D}(\mathrm{D}, \mathrm{n})^{3} \mathrm{He}$ reaction. The time-of-flight spectrometer TOFOR is mounted in the roof laboratory and views the plasma perpendicularly to the magnetic field [53]. The other two neutron emission spectrometers detect neutrons through reactions with a synthetic single-crystal diamond [54-56] and the liquid organic scintillator material NE213 [57], respectively. Both neutron emission spectrometers have compact designs and are mounted in the back of the magnetic proton recoil (MPR) deuterium-tritium neutron emission spectrometer (which is not used here). The lines-of-sight of the two compact neutron emission spectrometers are oblique forming an angle of $47^{\circ}$ with the core magnetic field.

The high-resolution HpGe GRS detector is mounted just behind TOFOR sharing the same line-of-sight $[16,58]$. Here we use measurements of the two competing two-step reactions ${ }^{9} \mathrm{Be}(\mathrm{D}, \mathrm{n} \gamma){ }^{10} \mathrm{~B}$ and ${ }^{9} \mathrm{Be}(\mathrm{D}, \mathrm{p} \gamma){ }^{10} \mathrm{Be}$. Beryllium is the first-wall surface material and therefore a major impurity at JET. It is also planned as first-wall surface material in ITER. Basic parameters of the reactions relevant to this study are summarized in table 1.

Figure 3 presents the NES measurements for the three spectrometers. The y-axes 
Table 1. Reactions producing the measured neutron and $\gamma$-ray spectra

\begin{tabular}{lll}
\hline Reaction & Energy release & Neutron or $\gamma$ energy \\
\hline $\mathrm{D}(\mathrm{D}, \mathrm{n})^{3} \mathrm{He}$ & $\mathrm{Q}=3.27 \mathrm{MeV}$ & $E_{n}=2.5 \mathrm{MeV}$ \\
${ }^{9} \mathrm{Be}(\mathrm{D}, \mathrm{n} \gamma)^{10} \mathrm{~B}$ & $\mathrm{Q}^{*}=(4.36-3.59) \mathrm{MeV}$ & $E_{\gamma}=2.868 \mathrm{MeV}$ \\
${ }^{9} \mathrm{Be}(\mathrm{D}, \mathrm{p} \gamma)^{10} \mathrm{Be}$ & $\mathrm{Q}^{*}=(4.59-3.37) \mathrm{MeV}$ & $E_{\gamma}=3.367 \mathrm{MeV}$ \\
\hline
\end{tabular}

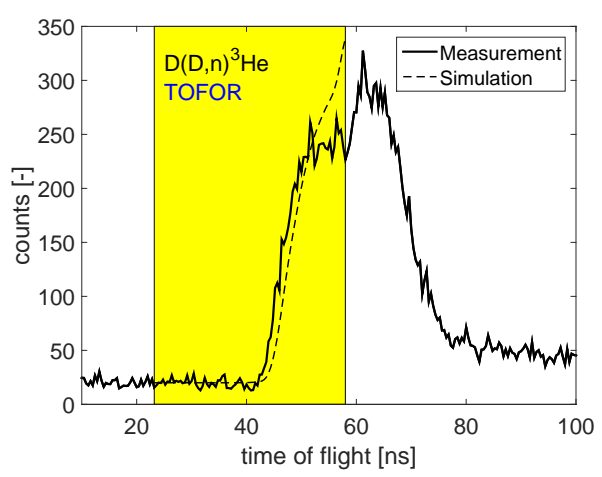

(a)

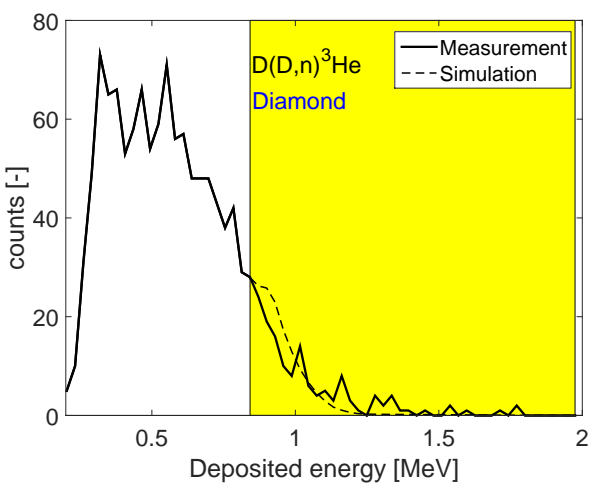

(b)

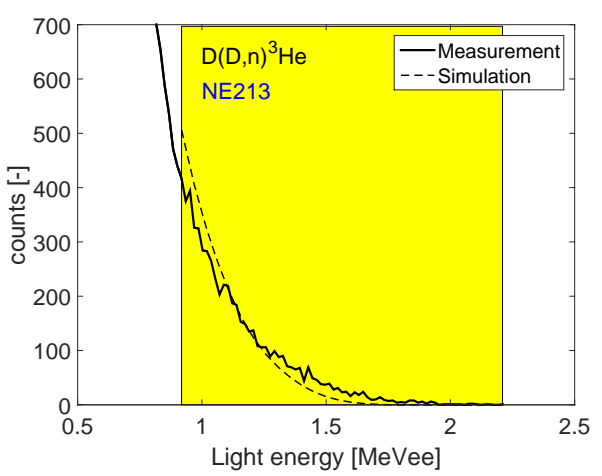

(c)

Figure 3. NES measurements in JET discharge \#86459 with three different neutron detectors together with synthetic measurements based on the ASCOT-RFOF code. The data used for the inversion is highlighted in yellow. (a) TOFOR. (b) Diamond. (c) Liquid scintillator NE213.

are the number of event counts per bin in all cases. However, the x-axes are given in units particular to each instrument as we will explain below. The instrumental units are related to the neutron energies via known instrumental response matrices which reflect that a recorded event could originate from neutrons in a wide range of possible energies. The determination of neutron energy spectra from the measurements is therefore an inverse problem in itself. We incorporated this inverse problem into the velocity-space tomography problem by formulating instrumental weight functions that include the instrumental response matrix and hence directly relate the instrumental units to velocity space $[28,59]$. The parts of the spectra that are used for the inversion are chosen based on weight functions and are highlighted in each case (see section 3 ). 
The measurements are shown together with synthetic measurements based on a numerical simulation with the ASCOT-RFOF code [24]. The absolute calibration of the measurements is comparatively uncertain. Hence we fitted the synthetic spectra to the measured spectra by multiplication with a single scaling factor per spectrum. This allows comparisons of the spectral shapes disregarding the absolute scaling.

TOFOR measures the distribution of times-of-flight of neutrons covering the distance between two detectors (figure $3(\mathrm{a})$ ). Ideally, the energy $E_{n}$ of an incoming neutron before scattering in the first detector can be related to the time-of-flight of the scattered neutrons between the two detectors by

$$
E_{n}=2 m_{n} \frac{R_{s}^{2}}{t_{t o f}^{2}}
$$

where $t_{t o f}$ is the time-of-flight, $m_{n}$ is the neutron mass and $R_{s}=0.705 \mathrm{~m}$ is the radius of the constant time-of-flight sphere on which both detectors are situated [53]. Plugging in the parameters leads to the handy formula for TOFOR

$$
E_{n} \approx\left(\frac{100 \mathrm{~ns}}{t_{\text {tof }}}\right)^{2} \mathrm{MeV} .
$$

However, the detailed instrumental response accounting for multiple scattering is more complicated as summarized in the instrumental response matrix [53,59] which we will account for. Equations 2 and 3 are useful because they describe the by far most likely response of TOFOR. Such a relation between the measured variable and the neutron energies does not exist for the diamond and the liquid scintillator spectrometers. We use the time-of-flight data between $22 \mathrm{~ns}$ and $58 \mathrm{~ns}$. Hardly any neutrons with timesof-flight below 42 ns are detected. Measurements of the absence of neutrons contain strong information about 2D velocity space (see section 3 ).

Figure 3(b) shows the measurements made with the single-crystal diamond detector. The detector measures distributions of energies deposited by neutrons in the diamond. In this case the instrumental response matrix reflects that neutrons in a wide range of possible energies above threshold energy can deposit a particular energy in the diamond $\left(E_{\text {dep }}<0.284 E_{n}\right)$ [59]. Here we use the data with deposited energies between $0.8 \mathrm{MeV}$ and $2 \mathrm{MeV}$ though hardly any detections are made above about $E_{\text {dep }}=1.4 \mathrm{MeV}$.

Figure 3(c) shows the measurements with the liquid scintillator NE213 detector. This detector is sensitive to neutron energies by measuring the emitted light produced when a neutron hits the scintillator material. The commonly used unit of this measurement is megaelectronvolt electron equivalent [MeVee] which is the energy of a hypothetical electron producing the emitted light. A particular flash of emitted light can again be produced by neutrons in a wide range of possible energies [59]. We use the data between 0.9 and 2.2 MeVee. Hardly any detections are made above about $2 \mathrm{MeVee.}$

Finally, the GRS measurements are made with a high-resolution HpGe detector, which can provide $1 \mathrm{keV}$ energy bins in the bandwidth of $10 \mathrm{MeV}$. Its fine instrumental response allows us to neglect it without loss of accuracy. The entire GRS data containing many $\gamma$-ray peaks is presented in figure 4 . The tiny highlighted regions show the spectral 
range that we use for velocity-space tomography. Figure 4 illustrates that measurement data from many other reactions can be exploited for tomographic inversion. We use the ${ }^{9} \mathrm{Be}(\mathrm{D}, \mathrm{n} \gamma){ }^{10} \mathrm{~B}$ and ${ }^{9} \mathrm{Be}(\mathrm{D}, \mathrm{p} \gamma){ }^{10} \mathrm{Be}$ reaction peaks as they are powerful and good cross section data are available. High-resolution views of these peaks near $2868 \mathrm{keV}$ and $3367 \mathrm{keV}$ are presented in figure 5. Even though the two peaks are recorded with the same detector, the velocity-space observation regions of these two spectra are different as we will discuss in the next section.

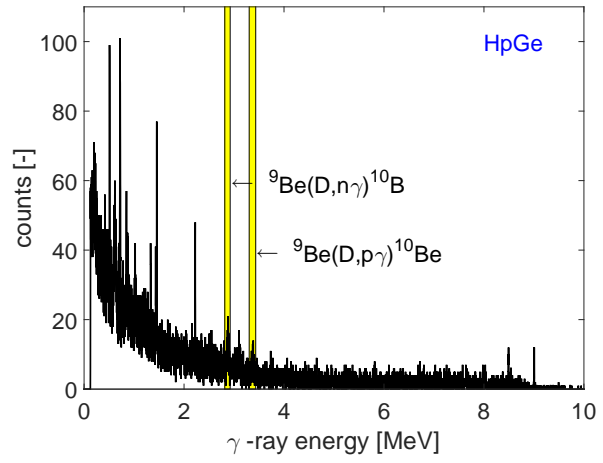

Figure 4. High-resolution GRS measurements showing the full $\gamma$-ray energy range of the HpGe detector. We use the high-resolution measurements of the two highlighted peaks. The spectral shapes of these two peaks are shown in figure 5 .

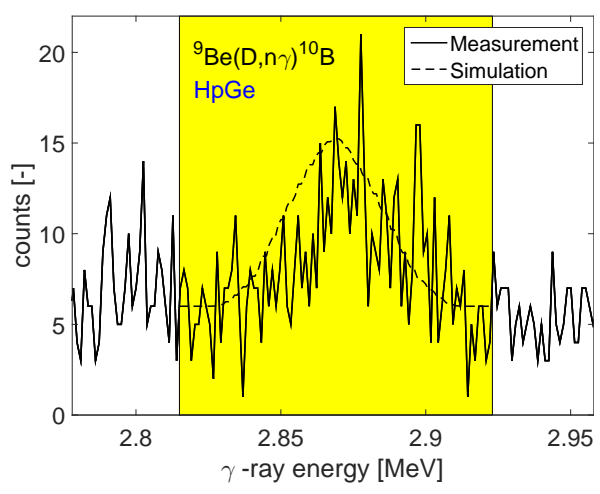

(a)

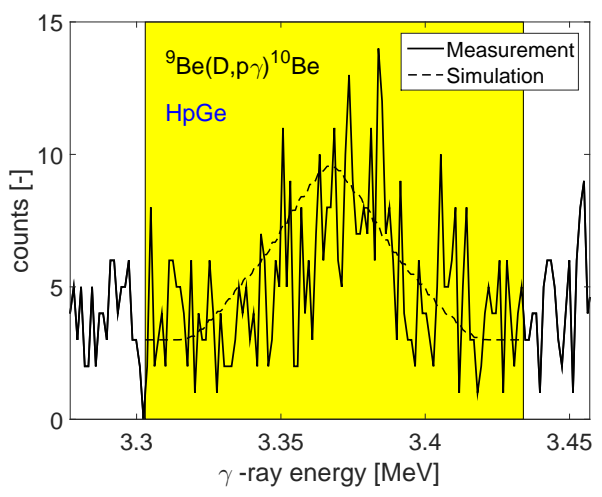

(b)

Figure 5. High-resolution GRS measurements of the spectral shapes of the two peaks used for the inversion together with synthetic measurements based on the ASCOTRFOF code. The data used for the inversion is highlighted in yellow. (a) The peak at $2868 \mathrm{keV}$ originates from the ${ }^{9} \mathrm{Be}(\mathrm{D}, \mathrm{n} \gamma)^{10} \mathrm{~B}$ reaction. (b) The peak at $3367 \mathrm{keV}$ originates from the ${ }^{9} \mathrm{Be}(\mathrm{D}, \mathrm{p} \gamma){ }^{10} \mathrm{Be}$ reaction.

\section{Instrumental weight functions}

Weight functions describe the velocity-space sensitivity of fast-ion diagnostics and have been calculated for FIDA [50,60], NPA [50], CTS [32], NES [28,29,59] and GRS [30,31]. 
NES and GRS weight functions including the instrumental response functions allow an efficient inversion of the measurements by velocity-space tomography. Weight functions $w$ connect the velocity distribution function $f$ to the measurement $S$ by

$$
S=\iint w f d v_{\|} d v_{\perp}
$$

In this section we emphasize two basic assumptions of the NES and GRS weight function formalism and illustrate the velocity-space interrogation regions of the various spectral measurements.

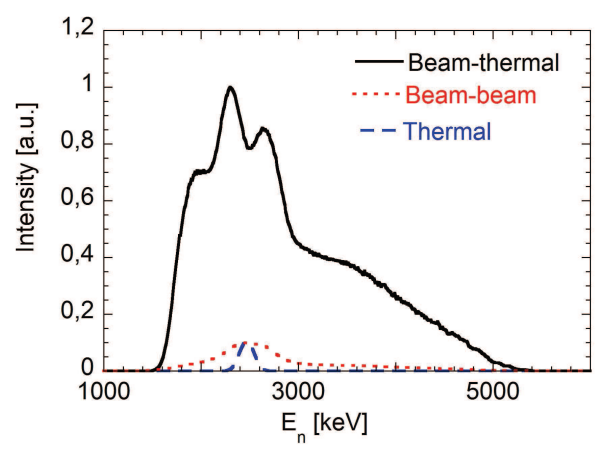

Figure 6. Relative contributions to the neutron energy spectra from beam-thermal, beam-beam and thermal reactions according to a GENESIS simulation of discharge \#86459. Beam-thermal reactions dominate over the other contributions.

First, NES and GRS weight functions rely on the assumption that most neutrons or $\gamma$ 's originate from a reaction between a fast ion and a thermal ion rather than between two thermal ions or two fast ions. These three basic types of reactions based on the reactant speeds are referred to as beam-beam, beam-thermal and thermal reactions. In JET discharge \#86459, beam-thermal reactions dominate (figure 6) as they often do. Beam-beam reactions are usually fairly unlikely due to the small number densities of fast particles whereas thermal reactions are usually fairly unlikely due to the small reaction cross sections at low energies. The beam-thermal assumption is always fulfilled for GRS involving ${ }^{9} \mathrm{Be}$ because the impurity is thermal and the reaction cross sections hence require large ion energies to allow reactions.

Second, here we neglect that the measurements are made in spatially different observation volumes. In principle, the neutrons and $\gamma^{\prime} s$ can originate from anywhere along the line-of-sight. However, most neutrons and $\gamma$ 's originate from the hot plasma core where the fusion reaction rates are largest as the inversion of neutron camera data shows (figure 7) [61]. Here we assume that all neutrons and $\gamma$ 's originate from the plasma core and neglect emissions from the periphery.

We now turn to the velocity-space interrogation regions of the measured data based on these assumptions. Figure 8 illustrates $5 \times 3$ weight functions representing the five detectors and three typical data points in the spectra for each detector. The amplitude of the weight functions is the measurable signal per ion in the relevant detector units (equation 4). Consequently, the white regions are not observable. The velocity-space 


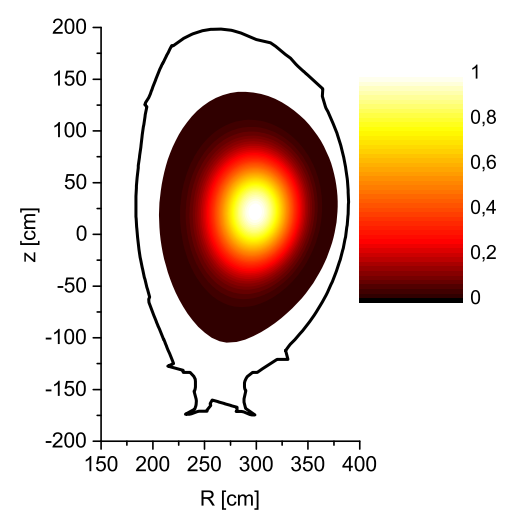

Figure 7. Measured neutron production rates [a.u.] obtained by inversion of neutron camera measurements (in position space) in discharge \#86459. Most neutrons are produced in the plasma core.

sensitivity (amplitudes) of the weight functions of the different spectral data points are rather different which suggests that the measurements complement each other well. In each case the weight function in the left column is sensitive to large portions of our target velocity space, i.e. the part of velocity space shown in figure 8 in which we seek to reconstruct the $2 \mathrm{D}$ velocity distribution function. The weight functions in the center column are sensitive to ions at intermediate and high energies but not to low-energy ions, and the weight functions in the right column are sensitive to high-energy ions only.

The data points for the inversion are selected using their associated weight functions. Here we do not seek to reconstruct the part of velocity space below $120 \mathrm{keV}$ since this low-energy region of velocity space is densely populated due to the NBI ions. The inclusion of this densely populated low-energy velocity-space region would make the inference of the relatively sparsely populated high-energy velocity-space region difficult. Hence we reject all data points associated with weight functions with significant values below $120 \mathrm{keV}$. In figure 8 the $120 \mathrm{keV}$ line is shown in blue. This method to reject the parts of the spectra that are sensitive to densely populated low-energy parts of velocity space is analogous to the rejection of the likewise very bright thermal halo feature originating from thermal ions in FIDA velocity-space tomography [60].

The weight functions covering only very high ion energies tend to be beneficial since the measured absence of signal for these weight functions helps finding empty regions of velocity space. Hence we use all weight functions reaching into the target velocity space. The rejected data at the high-energy end of the spectra are associated with weight functions that do not cover our target velocity space. 


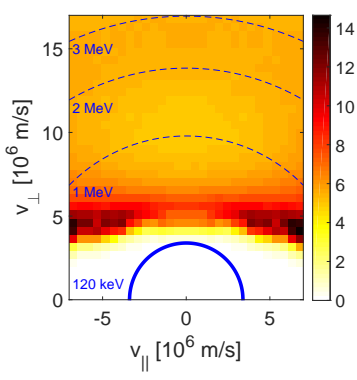

(a) $t_{t o f}=58 \mathrm{~ns}$

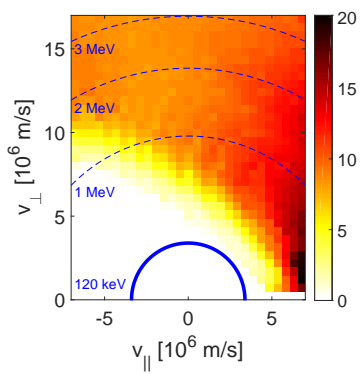

(d) $E_{d e p}=813 \mathrm{keV}$

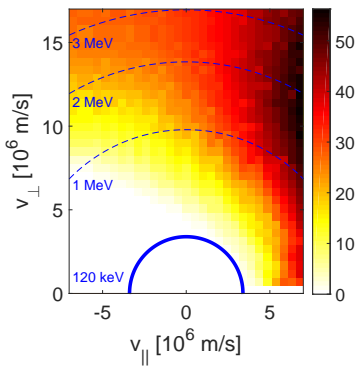

(g) $E_{p h}=0.9 \mathrm{MeVee}$

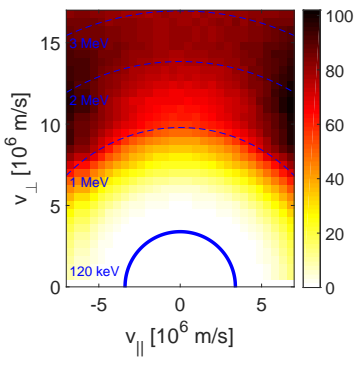

(j) $E_{\gamma}=2868 \mathrm{keV}$

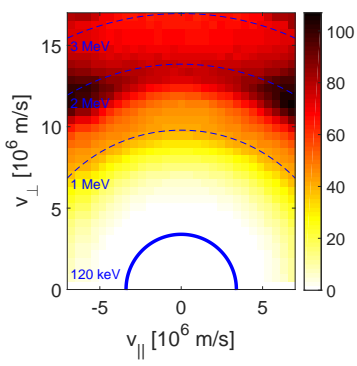

(m) $E_{\gamma}=3367 \mathrm{keV}$

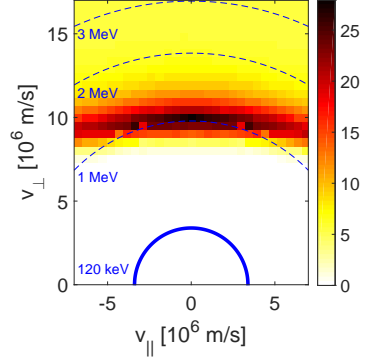

(b) $t_{t o f}=50 \mathrm{~ns}$

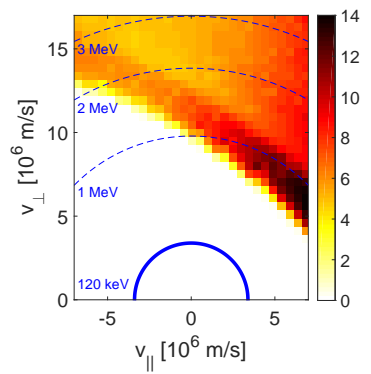

(e) $E_{d e p}=1016 \mathrm{keV}$

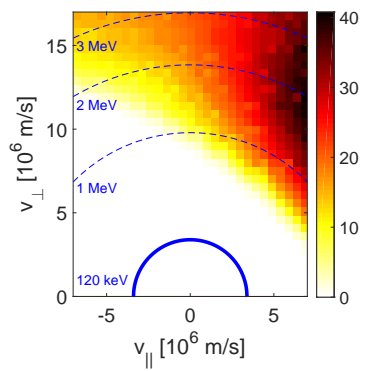

(h) $E_{p h}=1.2 \mathrm{MeVee}$

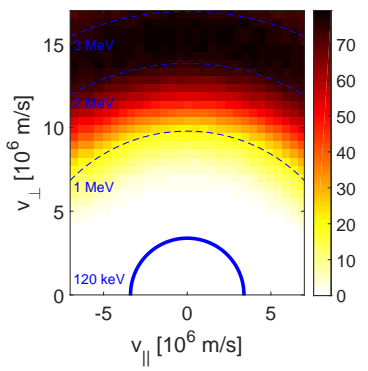

(k) $E_{\gamma}=2848 \mathrm{keV}$

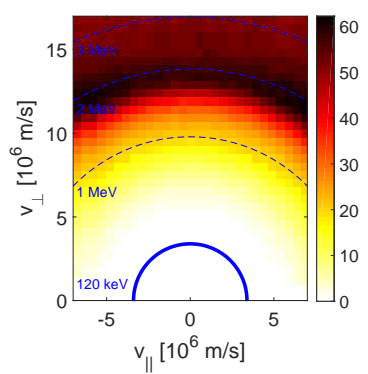

(n) $E_{\gamma}=3347 \mathrm{keV}$

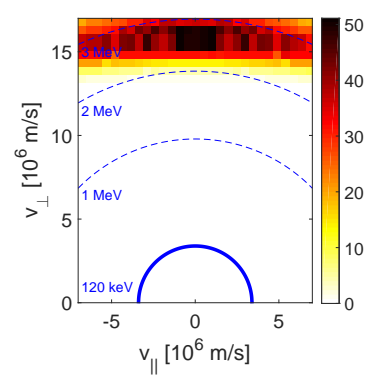

(c) $t_{t o f}=42 \mathrm{~ns}$

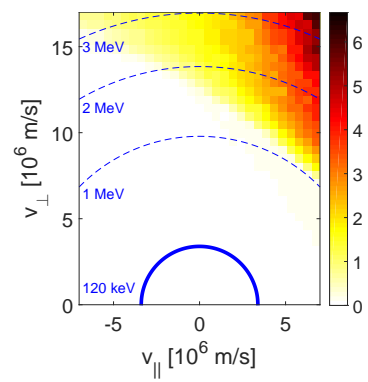

(f) $E_{d e p}=1191 \mathrm{keV}$

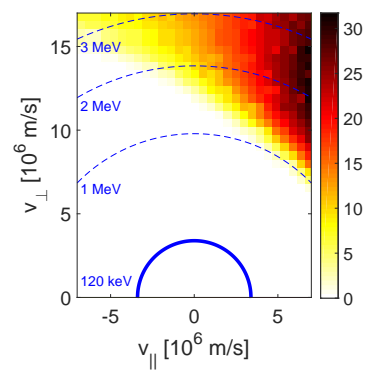

(i) $E_{p h}=1.5 \mathrm{MeVee}$

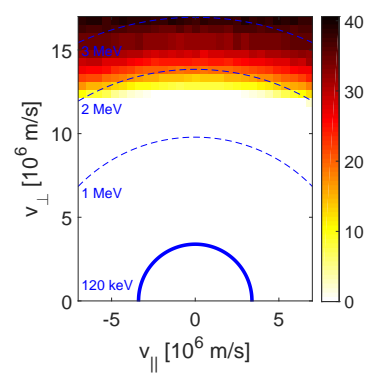

(l) $E_{\gamma}=2828 \mathrm{keV}$

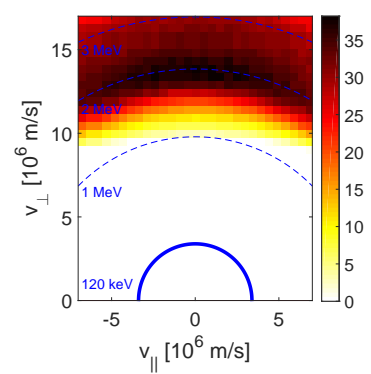

(o) $E_{\gamma}=3327 \mathrm{keV}$

Figure 8. Typical weight functions of the 5 detectors for low (left column), intermediate (center column), and high (right column) neutron or $\gamma$-ray energy shifts expressed in the particular diagnostic units. (a)-(c) TOFOR. (d)-(f) Diamond. (g)-(i) NE213. (j)-(l) HpGe $\gamma$-ray peak at $2868 \mathrm{keV}$. (m)-(o) HpGe $\gamma$-ray peak at $3367 \mathrm{keV}$. The blue dashed lines show $1 \mathrm{MeV}, 2 \mathrm{MeV}$ and $3 \mathrm{MeV}$, and the full blue line shows $120 \mathrm{keV}$. 


\section{MeV-range velocity-space tomography}

The NES and GRS weight functions allow us to pose the forward model for the computation of synthetic GRS and NES spectra efficiently as a matrix equation $[33,34]$

$$
S=W F
$$

which is the discretization of equation 4 . Here $S$ is a vector holding the measurements normalized by their uncertainties, $F$ is a vector holding the fast-ion velocity distribution function, and $W$ is a matrix composed of weight functions normalized by the uncertainty of the corresponding measurement [34]. As mentioned in section 2, the absolute scaling of the measured spectra is fairly uncertain. This uncertainty of the absolute scaling means that we will not compute absolutely scaled fast-ion velocity distribution functions. Here we calculate a constant scaling factor for each spectrum by fitting the synthetic spectra to the measured spectra and then scale all weight functions corresponding to a spectrum by this factor. This method provides a relative calibration of the five spectra, but does not change the spectral shapes.

The goal of velocity-space tomography is now to find $F$ (in a.u.), given $S$ and $W$. The matrix $W$ is ill-conditioned such that a useful and stable solution in $F$ cannot be computed without additional requirements on the solution. This is called regularization. Here we require that the gradients in $F$ are small and that $F$ is non-negative. Hence we pose the inverse problem using first-order Tikhonov regularization as the minimization problem [41]

$$
\text { minimize }\left\|\left(\begin{array}{c}
W \\
\lambda L
\end{array}\right) F-\left(\begin{array}{c}
S \\
0
\end{array}\right)\right\|_{2} \quad \text { subject to } F \geq 0
$$

which we solve using a standard non-negative least-squares algorithm [62]. The problem shown in expression 6 minimizes the sum of two-norm of the residual (upper row) and the two-norm of the additional constraint on the solution (lower row). Left-multiplication with the penalty matrix $L$ effects a finite difference approximation of a gradient [39]. The free regularization parameter $\lambda$ balances the smoothness requirement and the goodness of fit to the data.

\section{Benchmark of MeV-range velocity-space tomograghy and ASCOT-RFOF and SPOT-RFOF simulation}

This section demonstrates that measurements of $\mathrm{MeV}$-range 2D velocity distribution functions using velocity-space tomography are feasible and benchmarks the approach against numerical simulations with the ASCOT-RFOF [24] and SPOT-RFOF [26] codes. The simulated 2D velocity distribution functions averaged over the plasma core are presented in figures $9(\mathrm{a})$ and $9(\mathrm{c})$. The simulations of the formation of the fast-ion tail due to ICRF heating from the NBI seed population have been discussed previously $[24,26]$. The 2D velocity distribution functions show several expected features. The NBI population has energies below $120 \mathrm{keV}$. The ICRF waves draw a fast-ion tail in the 
$v_{\perp}$-direction $(>120 \mathrm{keV})$ since the wave electric field couples to this velocity component. The tail is close to symmetric about the $v_{\perp}$-axis. For third harmonic ICRF heating, the tail is expected to terminate at energies of about $2 \mathrm{MeV}$ since the wave-particle interaction becomes very weak above this energy. This strong decrease in the waveparticle interaction is also referred to as the barrier region [26]. The simulations predict that very few ions can be accelerated across the barrier region. Another feature of the simulations is that the tail width increases as $v_{\perp}$ decreases which is explained by collisions driving the distribution towards an isotropic distribution.

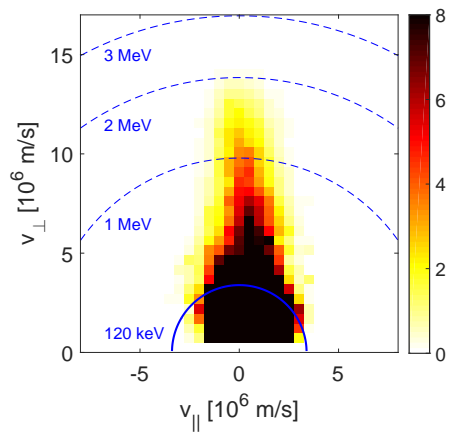

(a) ASCOT-RFOF

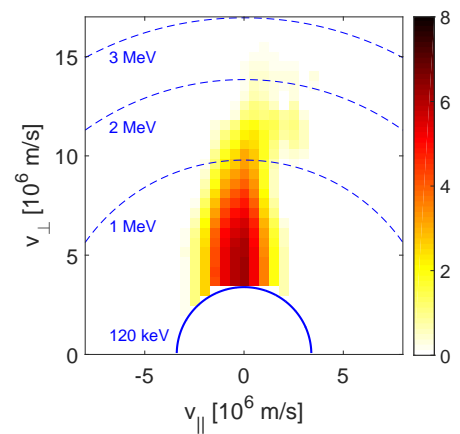

(b) Measurement

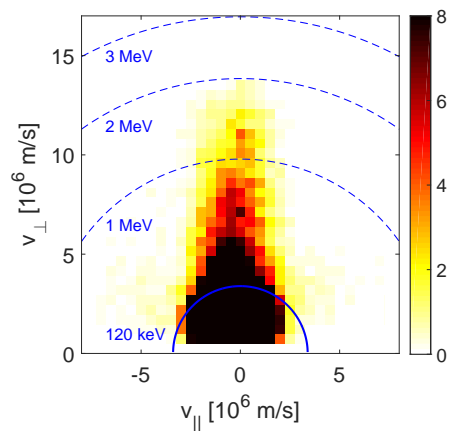

(c) SPOT-RFOF

Figure 9. Simulation with the ASCOT-RFOF and SPOT-RFOF codes and measurement of an $\mathrm{MeV}$-range ion velocity distribution function for third harmonic ICRF heating by velocity-space tomography. The dashed lines mark $1 \mathrm{MeV}, 2 \mathrm{MeV}$ and $3 \mathrm{MeV}$. The blue line marks $120 \mathrm{keV}$ which is the lower limit of our target velocity space.

Figure 9(b) shows the measurement of the core-averaged MeV-range 2D velocity distribution function based on the three NES spectra and the two GRS spectra. Several features of the inversion are in excellent agreement with the simulations. The measured fast-ion tail is close to symmetric about the $v_{\perp}$-axis as are the simulations. The predicted and measured tail lengths are very similar which confirms the presence of the barrier region at the predicted location. The measured width of the fast-ion tail including the widening for lower $v_{\perp}$ also follows the predictions very closely. Since the measurements are not absolutely calibrated, we can make no statement about the amplitudes. By construction the amplitudes of the simulation and the measurement are similar in arbitrary units.

The tomographic inversion allows the computation of derived quantities that are not experimentally accessible by other means. Here we integrate the $2 \mathrm{D}$ velocity distribution function over $v_{\|}$:

$$
f^{1 D}\left(v_{\perp}\right)=\int f\left(v_{\|}, v_{\perp}\right) d v_{\|} .
$$

Figure 10 shows a comparison of the measured and simulated $1 \mathrm{D}$ velocity distribution functions $f^{1 D}\left(v_{\perp}\right)$. As in figure 9, the agreement is excellent for $v_{\perp}>6 \times 10^{6} \mathrm{~m} / \mathrm{s}$. The inversion becomes uncertain for lower $v_{\perp}$ because the GRS weight functions do not 
reach into this region so that this low energy region is not well diagnosed.

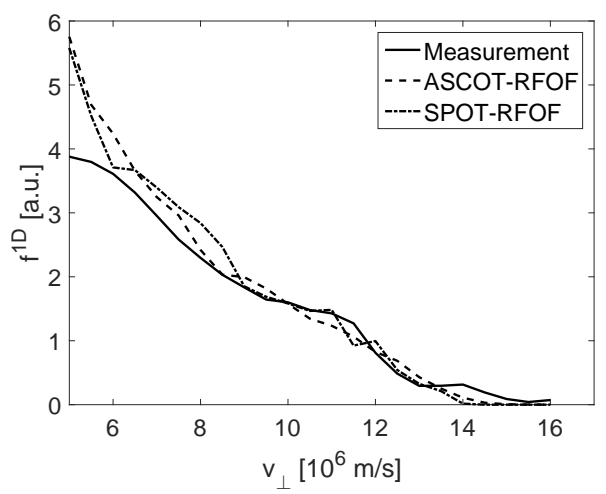

(a)

Figure 10. Comparison of measured and simulated 1D velocity distribution functions $f^{1 D}\left(v_{\perp}\right)$ illustrating the tail length in $v_{\perp}$ space.

Lastly, we note that the obtained results require the use of both NES and GRS diagnostics. We have computed inversions based on the three NES spectra alone and have not been able to find useful solutions. Figure 8 illustrates that the GRS measurements and TOFOR observe similar regions in velocity space whereas the oblique sightlines (i.e. the diamond and the liquid scintillator NES detectors) interrogate mostly $v_{\|}>0$. Hence the GRS measurements are essential to provide the necessary extra views for the region with $v_{\|}<0$. Further, the GRS weight functions have isolevels roughly following lines of constant energy whereas the weight functions of TOFOR have isolevels roughly following lines of constant $v_{\perp}$. This curvature of the GRS weight functions means that only GRS measurements observe the lower left corner of our target velocity space (low $v_{\perp}$ and $v_{\|}<0$ ). Thus the GRS diagnostic substantially improves the conditioning of the inverse problem, such that we can only obtain useful results by velocity-space tomography relying both on NES and GRS.

\section{Discussion}

In this section we discuss the potential and limitations of MeV-range velocity-space tomography. One limitation of our present method is that we do not have a standard candle to cross-calibrate the spectra without reference to a simulated fastion distribution function. This is different from FIDA velocity-space tomography since FIDA spectra are absolutely calibrated. Further, very strong direct beam emission is present in all FIDA spectra and can act as standard candle in each spectrum. Here we use a numerical simulation to cross-calibrate the measured spectra. An improvement could be to use an iterative procedure calibrating one spectrum against a synthetic spectrum calculated from a tomographic inversion based on the other four spectra. This would have the advantage that it does not rely on a simulation. An absolute scaling 
could be estimated after the inversion using the total neutron count rate. However, this is not attempted here.

A second limitation is that we neglect the spatial dimension of the problem. This limitation also appears to some extent in FIDA tomography as the measurement volumes of the different views are not exactly the same. A possible solution could be to formulate the NES and GRS weight functions with a spatial dimension along the line-of-sight which has already been done [29]. The downside of this idea for velocity-space tomography is that the spatial dimension increases the number of unknowns substantially. Thus more detectors providing more measurement data would be required. The $\gamma$-ray tomography provides spatial information, and it could be possible to combine the position-space and velocity-space tomography methods.

Possible applications of velocity-space tomography based on NES and GRS are not limited to JET, ITER and DEMO but include several present machines. Concerning ITER, our results hold promise that measurements of $\alpha$-particle $2 \mathrm{D}$ velocity distribution functions could be feasible. The ITER measurement requirements demand of the $\alpha$ particle density and the $\alpha$-particle energy spectrum [63] which can actually not be determined using traditional data analysis procedures. Velocity-space tomography allows core-averaged measurements of these parameters by integration of the measured 2D velocity-distribution function, provided that at least one diagnostic is absolutely calibrated. The $\alpha$-particles can be directly measured by CTS and GRS, and CTS measurements at ITER are being designed to be absolutely calibrated. $\alpha$-particles might further be detectable via knock-on collisions in NES spectra monitoring fuel ions. Deuterium and tritium 2D velocity distribution functions, as well as the derived fast-ion density and energy spectra, could also be determined by velocity-space tomography in ITER by NES and GRS.

The demonstration of a tomographic inversion of NES and GRS data will further be useful in many present tokamaks with NES and GRS diagnostics. For example, EAST has neutron spectrometers $[64,65]$ as well as two FIDA views [66]. ASDEX Upgrade has five FIDA views $[36,38,47,48]$, two CTS views [44-46] as well as NES [67,68] and GRS [69].

\section{Conclusions}

So far velocity-space tomography has focussed on FIDA data and been limited to energies below $120 \mathrm{keV}$ due to the low FIDA signal levels of more energetic ions. However, with burning plasmas on the horizon, the $2 \mathrm{D}$ velocity-space diagnostic of $\mathrm{MeV}$ range particles becomes essential to confront theories describing energetic particles. In this paper we extend the applicability of the velocity-space tomography method into the $\mathrm{MeV}$-range and demonstrate the measurement of a $2 \mathrm{D}$ velocity distribution function in a third harmonic ICRF heating scenario at JET. The tomographic inversion in the MeVrange velocity space requires that we base the inversion on NES and GRS measurements rather than on FIDA. The NES measurements are made with the time-of-flight detector 
TOFOR, a single-crystal diamond detector and a liquid scintillator detector based on the organic material NE213. The GRS measurements are made with a high-resolution high-bandwidth HpGe detector of which we use spectrally resolved data for two reaction peaks.

The tomographic inversion based on these five spectra is in excellent agreement with SPOT-RFOF and ASCOT-RFOF simulations which validates not only the velocityspace tomography approach but also the simulations. The measurement by velocityspace tomography confirms the predicted length and width of the fast-ion tail in velocity space. The measured tail length is consistent with the presence of the predicted barrier region in velocity space in which the wave-particle interaction is very weak. The measurement of an MeV-range 2D velocity distribution function based on GRS and NES at JET paves the way for measurements of $2 \mathrm{D} \alpha$-particle velocity distribution functions in the upcoming DT campaign at JET and at ITER. Finally, the ITER measurement requirements entail measurements of the $\alpha$-particle density and energy spectra. However, diagnostic weight functions suggest that these requirements cannot be fulfilled by individual diagnostics. Combined velocity-space tomography will allow measurements of these important parameters and is thus a promising diagnostic analysis tool for ITER.

\section{Acknowledgments}

We thank the ITPA Energetic Particle Physics Topical Group for its support. This work has been carried out within the framework of the EUROfusion Consortium and has received funding from the Euratom research and training programme 2014-2018 under grant agreement No 633053. The views and opinions expressed herein do not necessarily reflect those of the European Commission.

\section{References}

[1] Motojima O et al 2015 Nucl. Fusion 55104023

[2] Shimada M et al Progress in the ITER Physics Basis Chapter 1: Overview and Summary 2007 Nucl. Fusion 47 S1-S17

[3] Giruzzi G et al 2015 Nucl. Fusion 55073002

[4] Wenninger R et al 2015 Nucl. Fusion 55063003

[5] Keilhacker M et al 1999 Nucl. Fusion 39 209-234

[6] Heidbrink W and Sadler G 1994 Nucl. Fusion 34 535-615

[7] Jacquinot J et al ITER Physics Basis Chapter 5: Physics of energetic ions 1999 Nuclear Fusion 39 2471-2495

[8] Fasoli A et al Progress in the ITER Physics Basis Chapter 5: Physics of energetic ions 2007 Nucl. Fusion 47 S264-S284

[9] Sharapov S et al 2013 Nucl. Fusion 53104022

[10] Gorelenkov N, Pinches S and Toi K 2014 Nuclear Fusion 54125001

[11] Pinches S D et al 2015 Physics of Plasmas 22021807

[12] Chen L and Zonca F 2016 Rev. Mod. Phys. 88015008

[13] Romanelli F 2015 Nucl. Fusion 55104001 
[14] Kiptily V G et al 2010 Nucl. Fusion 50084001

[15] Murari A et al 2010 Rev. Sci. Instrum. 81 10E136

[16] Nocente M et al 2010 Rev. Sci. Instrum. 81 10D321

[17] Nocente M et al 2012 Nucl. Fusion 52063009

[18] Tardocchi M et al 2011 Physical Review Letters 107205002

[19] Gassner T et al 2012 Phys. Plasmas 19032115

[20] Shevelev A E et al 2013 Nucl. Fusion 53123004

[21] Hellesen C et al 2013 Nucl. Fusion 53113009

[22] Eriksson L G et al 1998 Nucl. Fusion 38 265-278

[23] Hellesen C et al 2010 Nucl. Fusion 50084006

[24] Eriksson J et al 2015 Nucl. Fusion 55123026

[25] Nocente M et al 2015 Nucl. Fusion 55123009

[26] Schneider M et al 2016 Nucl. Fusion 112022

[27] Salmi A et al 2006 Plasma Phys. Control. Fusion 48 717-726

[28] Jacobsen A S et al 2014 Rev. Sci. Instrum. 85 11E103

[29] Jacobsen A S et al 2015 Nucl. Fusion 55053013

[30] Salewski M et al 2015 Nucl. Fusion 55093029

[31] Salewski M et al 2016 Nucl. Fusion 56046009

[32] Salewski M et al 2011 Nucl. Fusion 51083014

[33] Salewski M et al 2012 Nucl. Fusion 52103008

[34] Salewski M et al 2013 Nucl. Fusion 53063019

[35] Salewski M et al 2014 Nucl. Fusion 54023005

[36] Geiger B et al 2015 Nucl. Fusion 55083001

[37] Salewski M et al 2015 Plasma Phys. Control. Fusion 57014021

[38] Weiland M et al 2016 Plasma Phys. Control. Fusion 58025012

[39] Jacobsen A S et al 2016 Plasma Phys. Control. Fusion 58045016

[40] Jacobsen A S et al 2016 Plasma Phys. Control. Fusion 58042002

[41] Salewski M et al 2016 Nucl. Fusion 56106024

[42] Rasmussen J et al 2016 Nucl. Fusion 56112014

[43] Jaulmes F et al 2016 Nucl. Fusion 56112012

[44] Salewski M et al 2010 Nucl. Fusion 50035012

[45] Nielsen S K et al 2015 Plasma Phys. Control. Fusion 57035009

[46] Rasmussen J et al 2015 Plasma Phys. Control. Fusion 57075014

[47] Geiger B et al 2011 Plasma Phys. Control. Fusion 53065010

[48] Geiger B et al 2015 Plasma Phys. Control. Fusion 57014018

[49] Heidbrink W W et al 2004 Plasma Phys. Control. Fusion 46 1855-1875

[50] Heidbrink W W et al 2007 Plasma Phys. Control. Fusion 49 1457-1475

[51] Heidbrink W W 2010 Rev. Sci. Instrum. 81 10D727

[52] Hirvijoki E et al 2014 Comp. Phys. Comm. 185 1310-1321

[53] Gatu Johnson M et al 2008 Nucl. Inst. Meth. Phys. Res. A 591 417-430

[54] Cazzaniga C et al 2014 Review of Scientific Instruments 85043506

[55] Nocente M et al 2015 Review of Scientific Instruments 86103501

[56] Muraro A et al 2016 Review of Scientific Instruments 87 11D833

[57] Binda F et al 2014 Rev. Sci. Instrum. 85 11E123

[58] Nocente M et al 2013 IEEE Transactions on Nuclear Science 60 1408-1415

[59] Jacobsen A S et al 2017 submitted

[60] Salewski M et al 2014 Plasma Phys. Control. Fusion 56105005

[61] Nocente $\mathrm{M}$ et al 2016 J. Inst. 11 C03001-C03001

[62] Lawson C and Hanson R 1974 Solving Least Squares Problems (Prentic-Hall, Englewood Cliffs, NJ)

[63] Donné A J H et al Progress in the ITER Physics Basis Chapter 7: Diagnostics 2007 Nucl. Fusion 


\section{S337-S384}

[64] Yuan X et al 2013 Journal of Instrumentation 8 P07016-P07016

[65] Zhang X et al 2014 Nuclear Fusion 54104008

[66] Huang J et al 2014 Rev. Sci. Instrum. 85 11E407

[67] Giacomelli L et al 2011 Rev. Sci. Instrum. 82123504

[68] Tardini G et al 2012 Journal of Instrumentation 7 C03004-C03004

[69] Nocente M et al 2012 Nucl. Fusion 52094021 